\title{
Prognostic significance of thymidylate synthase expression in pancreatic adenocarcinoma: A meta-analysis
}

\author{
YAN-MEI GUO $^{1^{*}}$, MIN ZHU ${ }^{2}$ and WEI-WEI YU ${ }^{3 *}$ \\ ${ }^{1}$ Department of Gastroenterology, Shanghai Tenth People's Hospital of Tongji University, Shanghai 200072; ${ }^{2}$ Department of \\ Biostatistics, School of Public Health, Fudan University, Shanghai 200032; ${ }^{3}$ Department of Radiation Oncology, \\ Shanghai Sixth People's Hospital Affiliated to Jiao Tong University, Shanghai 200233, P.R. China
}

Received July 31, 2014; Accepted September 2, 2014

DOI: $10.3892 /$ mco. 2014.443

\begin{abstract}
The prognostic significance of thymidylate synthase (TS) overexpression in pancreatic adenocarcinoma has been extensively investigated; however, data on the survival of patients with pancreatic adenocarcinoma remain equivocal. We performed a meta-analysis of previous studies to assess the effects of TS overexpression on the overall survival (OS) of patients with pancreatic adenocarcinoma, using hazard ratio (HR) with its $95 \%$ confidence interval (CI). A total of 5 studies, including 425 patients, were subjected to the final analysis. The pooled HR was 0.72 (95\% CI: $0.41-1.25 ; \mathrm{Z}=1.18$, $\mathrm{P}=0.238$ ), indicating that TS expression exerted no significant survival effect on patients with pancreatic adenocarcinoma. The combined HR was 0.46 (95\% CI: 0.31-0.68; $\mathrm{Z}=3.95$, $\mathrm{P}<0.001)$, limiting the analysis to the studies assessing $\mathrm{R} 0$ resection patients, which indicated that a high expression of TS was significantly correlated with better OS in patients with pancreatic adenocarcinoma who underwent $\mathrm{R} 0$ resection. This meta-analysis identified TS as an independent factor predicting favourable outcome following R0 curative resection in patients with pancreatic adenocarcinoma.
\end{abstract}

\section{Introduction}

Pancreatic adenocarcinoma is the fourth most common cause of cancer-related mortality worldwide (1). Despite recent advances in therapy, the overall survival (OS) of patients with pancreatic adenocarcinoma remains dismal. Radical surgery, particularly $\mathrm{R} 0$ resection, offers the only chance for cure or long-term survival. However, the 5-year survival rate in

Correspondence to: Dr Wei-Wei Yu, Department of Radiation Oncology, Shanghai Sixth People's Hospital Affiliated to Jiao Tong University, 600 Yi Shan Road, Xu Hui District, Shanghai 200233, P.R. China

E-mail: yww333@126.com

*Contributed equally

Key words: pancreatic adenocarcinoma, thymidylate synthase, survival, meta-analysis patients with pancreatic adenocarcinoma remains $<20 \%$, even following radical resection (2-4). Therefore, surgery alone is clearly not an adequate approach to achieving long-term survival in patients with pancreatic adenocarcinoma. Effective adjuvant therapy may exert a potentially significant effect on OS. 5-Fluorouracil (5-FU) is widely used in the treatment of gastrointestinal tumors, including pancreatic adenocarcinoma. Thymidylate synthase (TS), a rate-limiting enzyme for DNA synthesis, is the target of 5-FU. Following its metabolism, 5-FU may bind TS, thereby preventing binding of its normal substrate, dUMP, thus inhibiting DNA synthesis (5). The available TS pool may, therefore, be of interest in the response to chemotherapy. Previous clinical studies have demonstrated that TS expression level predicts the response to fluorouracil-based chemotherapy in several types of cancer (6-8).

The value of TS in determining whether a patient may benefit from fluorouracil-based adjuvant therapy has also been investigated in pancreatic cancer. However, conflicting results have been reported from various laboratories. Besides its predictive value, the reported findings on the expression levels and prognostic value of TS are also inconsistent (9-13). Therefore, a meta-analysis was performed to assess the prognostic value of TS protein expression for the survival of patients with pancreatic adenocarcinoma.

\section{Materials and methods}

Search strategy and selection criteria. The MEDLINE database was searched online in March, 2013 for bibliographic information regarding studies on TS expression status and survival in patients with pancreatic adenocarcinoma. The following MESH headings, keywords and text words were used: i) pancreatic and neoplasm OR cancer OR carcinoma OR adenocarcinoma; and ii) thymidylate synthase OR TS. The reference lists of the retrieved articles and previous narrative reviews were scanned for additional potentially relevant articles.

Studies were included in the meta-analysis if they met the following criteria: i) expression of TS was evaluated in the tumor tissue as opposed to serum or metastatic tissue, or in tissue adjacent to the tumour; ii) the expression of TS protein was measured by immunohistochemistry (IHC) or enzyme-linked immunosorbent assay (ELISA); iii) analysis of the association 
Table I. Main characteristics and results of the eligible studies.

\begin{tabular}{|c|c|c|c|c|c|c|c|c|}
\hline First author & Year & Method & Specimen & Antibody & $\begin{array}{l}\text { High TS } \\
\text { expression } \\
\text { no./total }(\%)\end{array}$ & Margins & $\begin{array}{c}\text { TS effect } \\
\text { on OS }\end{array}$ & (Refs.) \\
\hline van der Zee & 2012 & $\mathrm{IHC}$ & Paraffin & $\begin{array}{l}\text { Anti-TS TS106 mAb, } \\
\text { Dako Netherlands BV } \\
\text { (Heverlee, Belgium) }\end{array}$ & $24 / 59(40.7)$ & R0 & $\mathrm{S}$ (better) & (9) \\
\hline \multirow[t]{2}{*}{ Kondo } & 2011 & $\mathrm{IHC}$ & Paraffin & Anti-TS pAb & $68 / 106(64.2)$ & $\mathrm{R} 0 / \mathrm{R} 1$ & NS & (10) \\
\hline & & & & Taiho Pharmaceutical (Tokyo, Japan) & & & & \\
\hline Nakahara & 2010 & ELISA & Frozen & $\begin{array}{c}{\left[6-{ }^{3} \mathrm{H}\right]-\text { FdUMP binding assay, }} \\
\text { Moravek Biochemicals, Inc. } \\
\text { (Brea, CA, USA) }\end{array}$ & $6 / 18(33.3)$ & R0 & NS & (11) \\
\hline $\mathrm{Hu}$ & 2003 & $\mathrm{IHC}$ & Paraffin & $\begin{array}{c}\text { Anti-TS TS106 mAb, } \\
\text { NeoMarkers, Inc. (Fremont, CA, USA) }\end{array}$ & $83 / 132(62.9)$ & $\mathrm{R} 0 / \mathrm{R} 1$ & $\mathrm{~S}$ (worse) & (13) \\
\hline Takamura & 2002 & IHC & Paraffin & $\begin{array}{c}\text { Anti-TS pAb, } \\
\text { Taiho Pharmaceutical (Tokyo, Japan) }\end{array}$ & 49/110 (44.5) & $\mathrm{R} 0 / \mathrm{R} 1$ & S (better) & (19) \\
\hline Takamura & 2002 & $\mathrm{IHC}$ & Paraffin & $\begin{array}{c}\text { Anti-TS pAb, } \\
\text { Taiho Pharmaceutical (Tokyo, Japan) }\end{array}$ & $31 / 72(43.1)$ & R0 & $\mathrm{S}$ (better) & $(19)$ \\
\hline
\end{tabular}

TS, thymidylate synthase; OS, overall survival; IHC, immunohistochemistry; S, significant; NS, non-significant; ELISA, enzyme-linked immunosorbent assay; mAb, monoclonal antibody; pAb, polyclonal antibody.

between TS expression and OS; and iv) studies were published as full-text in English. Manual selection of relevant studies was performed based on the summary analysis.

Reviews and conference abstracts were excluded due to limited data for evaluation. When multiple articles pertained to overlapping populations of patients, only the newest, largest, or most informative single article was selected.

Data extraction. Data was carefully extracted independently by two investigators (Guo and Zhu) and controversies were resolved through discussion according to the abovementioned criteria. If an agreement could not be reached, an expert was invited to the discussion. The following information was extracted from each study: first author, year of publication, method, specimen, number of eligible patients, antibody, resection and OS results.

The effect of TS expression on OS was estimated for each study by the hazard ratio (HR) with its $95 \%$ confidence interval (CI), according to the methods described by Tierney et al (14). In summary, when the estimated HR and its standard error were described in the publications, we obtained these values directly; when these statistical variables were not provided explicitly in an article, they were calculated directly using two of the following parameters: the CI for the HR, the log-rank statistic, its $\mathrm{P}$-value or the O-E statistic (difference between numbers of observed and expected events); when those data were not available, the following were investigated: total number of events, number of patients at risk in each group and the log-rank statistic or its P-value, allowing calculation of an approximation of the HR estimate; when the only available data were in the form of graphical representations, they were calculated from Kaplan-Meier survival curves. The Kaplan-Meier curves were read by two investigators using
Engauge Digitizer software, version 4.1 (Mark Mitchell, Boston, MA, USA) independently to reduce inaccuracy in extracted survival rates.

Statistical analysis. A $\chi^{2}$ test-based Q statistic test for between-study heterogeneity was used with a P-value of 0.1 rather than 0.05 to determine statistical significance (15). $\mathrm{P}>0.10$ indicated a lack of heterogeneity among studies, so the fixed-effects model was used to combine HR. Otherwise, the random-effects model was used (16). By convention, the effect of TS on OS was considered as statistically significant if the $95 \%$ CI for the overall HR did not overlap 1. Evidence of publication bias was evaluated by the funnel plot with Begg's test (17) and Egger's linear regression asymmetry test (18). For these analyses, $\mathrm{P}<0.05$ was considered representative of statistically significant publication bias. All the statistical analyses were performed by STATA 12.0 software (StataCorp, College Station, TX, USA).

\section{Results}

Study characteristics. The characteristics of the eligible studies are summarized in Table I. A total of 5 studies $(9-11,13,19)$, published between 2002 and 2012, met the inclusion criteria for this review. A total of 425 patients were subjected to the final analysis. All the studies were based on the data of retrospective analysis. Among the 5 studies investigating OS, a significant association between TS high expression and OS was identified in 3, including 2 associating TS high expression with better OS and 1 associating TS high expression with worse OS. The remaining 2 studies yielded negative results.

The expression of TS was measured by IHC in 4 of the 5 studies and by ELISA in the remaining study. The IHC 


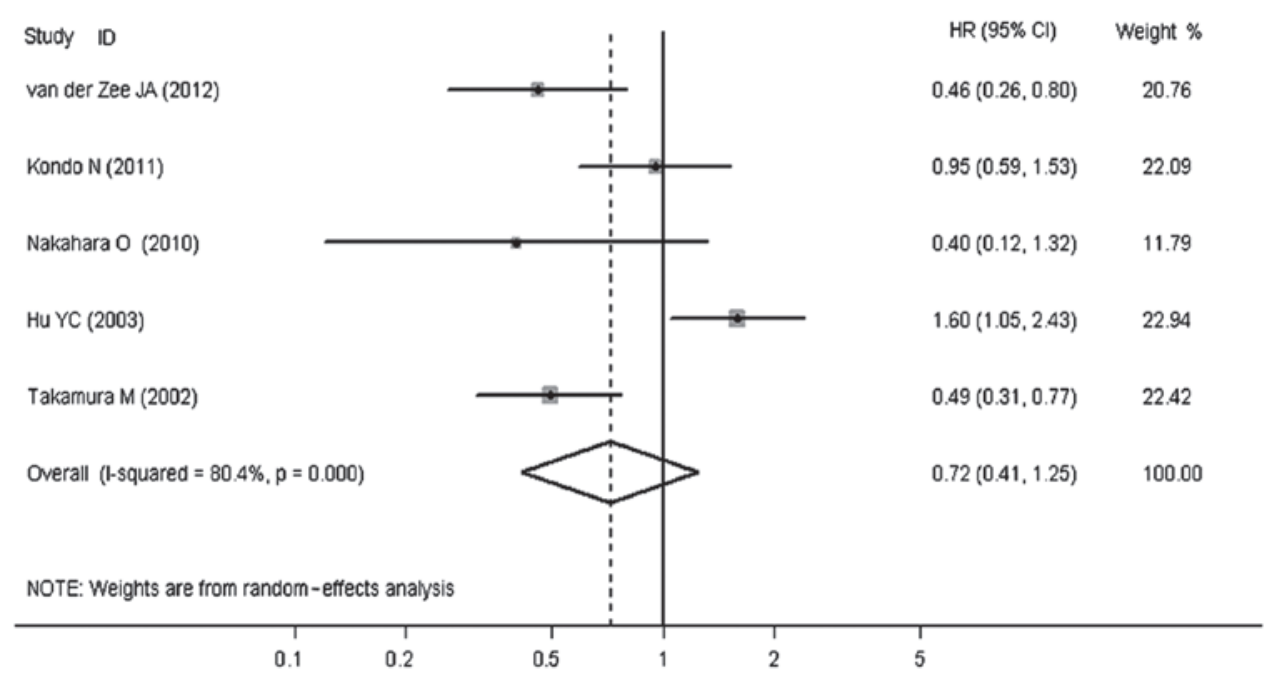

Figure 1. Forest plot of HR for 5 studies assessing the association of thymidylate synthase expression with overall survival in pancreatic adenocarcinoma. HR, hazard ratio; $\mathrm{CI}$, confidence interval.

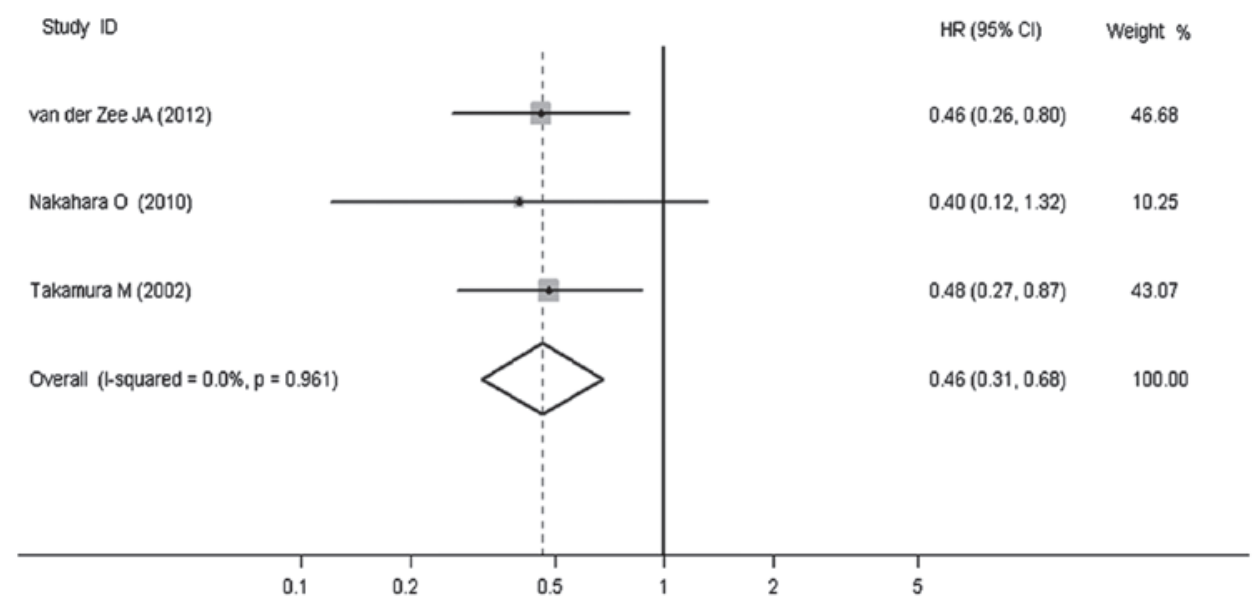

Figure 2. Forest plot of HR for the 3 studies assessing R0 resection in pancreatic adenocarcinoma. HR, hazard ratio; CI, confidence interval.

techniques used varied widely among the studies, with a wide range of dilutions $(1: 15-1: 1,000)$. According to the cut-off values for TS high expression as defined by the authors of each study, a total of 230 patients $(54.1 \%)$ in this meta-analysis exhibited high TS expression, with a range of $25-75 \%$.

Three studies accrued patients who had received R0 and $\mathrm{R} 1$ resection $(10,13,19)$ and stratified results regarding the association between TS expression with OS for resection type (R0 or R1) were only mentioned in 1 study (19). Only the patients who underwent $\mathrm{R} 0$ resection were analyzed in the remaining 2 studies $(9,11)$.

Correlation of TS expression and OS. The pooled HR was 0.72 (95\% CI: $0.41-1.25 ; \mathrm{Z}=1.18, \mathrm{P}=0.238$ ), indicating that TS expression exerted no significant survival effect on patients with pancreatic adenocarcinoma. Heterogeneity testing revealed that there was inter-study heterogeneity $(\mathrm{Q}=20.46$, $\mathrm{P}<0.001$ ). (Fig. 1). When we limited the analysis to the studies assessing R0 resection patients, the combined HR was 0.46 (95\% CI: 0.31-0.68; $\mathrm{Z}=3.95, \mathrm{P}<0.001$ ), without any evidence of heterogeneity $(\mathrm{Q}=0.08, \mathrm{P}=0.961)$, which indicated that a high expression of TS was significantly correlated with better OS in patients with pancreatic adenocarcinoma who underwent R0 resection (Fig. 2).

Publication bias statistics obtained by the methods of Begg and Mazumdar (17) and Egger et al (18) were as follows: all 5 eligible studies $(\mathrm{P}=0.806$ and $\mathrm{P}=0.414)$; and the 3 studies assessing $\mathrm{R} 0$ resection $(\mathrm{P}=1.00$ and $\mathrm{P}=0.279)$. This suggested absence of publication bias in all the studies.

\section{Discussion}

In the present meta-analysis, a total of 5 eligible studies, including 425 patients, were finally analyzed to investigate the OS of patients with pancreatic adenocarcinoma according to the expression of TS. Our results suggested that TS expression exerted no significant effect on OS, with a combined OR of 0.72 (95\% CI: 0.41-1.25). Of note, we observed a significant correlation between TS high expression and a better prognosis in patients with $\mathrm{R} 0$ resectable pancreatic adenocarcinoma, with a combined HR of 0.46 (95\% CI: 0.31-0.68). Our results, suggesting that TS positively affects prognosis in $\mathrm{R} 0$ resectable 
pancreatic adenocarcinoma, are comparable to those obtained by Takemura et al (19). However, this effect was not observed in all the studies. The study of Hu et al (13) identified high TS expression as an independent predictor of poor prognosis. This difference in effect on outcome may be attributed to the R1 cases included in that study, since Takemura et al (19) also observed a different effect between resectable and unresectable disease.

As we performed a meta-analysis, we had to address heterogeneity issues; significant heterogeneity was detected among the 5 studies in our analysis. When analysis was limited to the 3 studies assessing R0 resection patients, the heterogeneity disappeared. Therefore, resection type may not only be a major cause of heterogeneity, but also a decisive factor in the biological effect of TS expression.

Factors associated with immunostaining may also cause heterogeneity. In the present meta-analysis, 4 studies performing IHC staining were included. IHC is currently the most commonly used method, as it is easy to apply, cost-effective and able to detect changes in a low proportion of tumour cells on a number of samples and formalin-fixed tissues for retrospective studies. However, a certain variation in methodological factors, such as different primary antibodies, a wide range of dilutions and cut-off points from arbitrary choices by investigators, contributed to a wide range of positive protein expression. In this study, the value of high TS expression was ranged $25-75 \%$, which may have contributed to the observed heterogeneity. Therefore, additional, more precise methods are required to validate the IHC results, such as molecular biology techniques.

Publication bias (20) is a well known problem in meta-analyses. Positive results tend to be accepted by journals, whereas negative results are often rejected or not even submitted. However, our analysis did not identify publication bias; therefore, the summary statistics obtained may approximate the actual average.

Certain limitations of this meta-analysis should be taken into consideration when interpreting the findings. First, we restricted our analysis to studies published in English and the majority of small studies that met the eligibility criteria were excluded based on language criteria, which may have led to an overestimation of effect sizes (21). Second, although a meta-analysis based on individual data is considered to be the gold standard, we used meta-analysis based on literature in this study, as individual patient data were difficult to access in different studies. Finally, the number of published studies was not adequate for a comprehensive analysis.

In conclusion, our meta-analysis suggests that TS expression may represent a strong candidate predictive biomarker for survival in patients with resectable pancreatic adenocarcinoma, particularly those undergoing R0 resection. In view of some of the limitations of this meta-analysis, larger studies, using standardized unbiased methods and enrolling participants worldwide, with more detailed individual data, are required. Furthermore, the predictive value of TS in pancreatic adenocarcinoma requires confirmation by finely designed prospective studies or clinical trails.

\section{Acknowledgements}

This study was funded by a research grant (no. 20124246) from the Scientific Research Found Projects of Shanghai
Health Bureau and a research grant (no. 81201883) from the National Nature Science Foundation of China.

\section{References}

1. Jemal A, Siegel R, Xu J and Ward E: Cancer statistics, 2010. CA Cancer J Clin 60: 277-300, 2010.

2. Shimada K, Sakamoto Y, Sano T and Kosuge T: Prognostic factors after distal pancreatectomy with extended lymphadenectomy for invasive pancreatic adenocarcinoma of the body and tail. Surgery 139: 288-295, 2006.

3. Wagner M, Redaelli C, Lietz M, Seiler CA, Friess H and Buchler MW: Curative resection is the single most important factor determining outcome in patients with pancreatic adenocarcinoma. Br J Surg 91: 586-594, 2004.

4. Li D, Xie K, Wolff R and Abbruzzese JL: Pancreatic cancer. Lancet 363: 1049-1057, 2004.

5. Longley DB, Harkin DP and Johnston PG: 5-fluorouracil: mechanisms of action and clinical strategies. Nat Rev Cancer 3: 330-338, 2003

6. Aguiar S Jr, Lopes A, Soares FA, Rossi BM, et al: Prognostic and predictive value of the thymidylate synthase expression in patients with non-metastatic colorectal cancer. Eur J Surg Oncol 31: 863-868, 2005.

7. Edler D, Glimelius B, Hallström M, et al: Thymidylate synthase expression in colorectal cancer: a prognostic and predictive marker of benefit from adjuvant fluorouracil-based chemotherapy. J Clin Oncol 20: 1721-1728, 2002.

8. Formentini A, Henne-Bruns D and Kornmann M: Thymidylate synthase expression and prognosis of patients with gastrointestinal cancers receiving adjuvant chemotherapy: a review. Langenbecks Arch Surg 389: 405-413, 2004.

9. van der Zee JA, van Eijck CH, Hop WC, et al: Expression and prognostic significance of thymidylate synthase (TS) in pancreatic head and periampullary cancer. Eur J Surg Oncol 38: 1058-1064, 2012.

10. Kondo N, Murakami Y, Uemura K, et al: Prognostic impact of dihydropyrimidine dehydrogenase expression on pancreatic adenocarcinoma patients treated with S-1-based adjuvant chemotherapy after surgical resection. J Surg Oncol 104: 146-154, 2011.

11. Nakahara $\mathrm{O}$, Takamori $\mathrm{H}$, Tanaka $\mathrm{H}$, et al: Clinical significance of dihydropyrimidine dehydrogenase and thymidylate synthase expression in patients with pancreatic cancer. Int J Clin Oncol 15: 39-45, 2010.

12. Shimoda M, Sawada T and Kubota K: Thymidylate synthase and dihydropyrimidine dehydrogenase are upregulated in pancreatic and biliary tract cancers. Pathobiology 76: 193-198, 2009.

13. Hu YC, Komorowski RA, Graewin S, et al: Thymidylate synthase expression predicts the response to 5-fluorouracil-based adjuvant therapy in pancreatic cancer. Clin Cancer Res 9: 4165-4171,2003.

14. Tierney JF, Stewart LA, Ghersi D, Burdett S and Sydes MR: Practical methods for incorporating summary time-to-event data into meta-analysis. Trials 8: 16, 2007.

15. Lau J, Ioannidis JP and Schmid CH: Quantitative synthesis in systematic reviews. Ann Intern Med 127: 820-826, 1997.

16. DerSimonian R and Laird N: Meta-analysis in clinical trials. Control Clin Trials 7: 177-188, 1986.

17. Begg CB and Mazumdar M: Operating characteristics of a rank correlation test for publication bias. Biometrics 50: 1088-1101, 1994.

18. Egger M, Davey Smith G, Schneider M and Minder C: Bias in meta-analysis detected by a simple, graphical test. BMJ 315: 629-634, 1997.

19. Takamura M, Nio Y, Yamasawa K, Dong M, Yamaguchi K and Itakura M: Implication of thymidylate synthase in the outcome of patients with invasive ductal carcinoma of the pancreas and efficacy of adjuvant chemotherapy using 5-fluorouracil or its derivatives. Anticancer Drugs 13: 75-85, 2002.

20. Begg CB and Berlin JA: Publication bias: A problem in interpreting medical data. J R Stat Soc Ser A 151: 419-463, 1988.

21. Pham B, Klassen TP, Lawson ML and Moher D: Language of publication restrictions in systematic reviews gave different results depending on whether the intervention was conventional or complementary. J Clin Epidemiol 58: 769-776, 2005. 